\title{
Influence of renal function on stroke outcome after mechanical thrombectomy: a prospective cohort study
}

\author{
Xiding Pan ${ }^{1,2}$, Feng Zhou ${ }^{2}$, Rui Shen ${ }^{2}$, Yubing Zhu', Hisatomi Arima ${ }^{3}$, Jie Yang ${ }^{4 \dagger}$ and Junshan Zhou ${ }^{2^{*+}}$
}

\begin{abstract}
Background: For acute ischemic stroke (AIS) patient receiving mechanical thrombectomy (MT), renal dysfunction was an independent risk factor of contrast-induced nephropathy which may affect clinical outcomes. However, the influence of renal function on stroke outcomes is still controversial. Thus, we aim to investigate the association between renal function and outcomes of AIS patients receiving MT.

Methods: All consecutive stroke patients receiving MT were included in a prospective stroke registry in China from April 2015 to February 2019. Estimated glomerular filtration rate (eGFR) was measured on admission and categorized into G1 $\left(\geq 90 \mathrm{ml} / \mathrm{min} / 1.73 \mathrm{~m}^{2}\right)$, G2 $\left(60-89 \mathrm{ml} / \mathrm{min} / 1.73 \mathrm{~m}^{2}\right), \mathrm{G} 3 \mathrm{a}\left(45-59 \mathrm{ml} / \mathrm{min} / 1.73 \mathrm{~m}^{2}\right)$ and G3b-5 $\left(\leq 44 \mathrm{ml} / \mathrm{min} / 1.73 \mathrm{~m}^{2}\right)$. Multivariable logistic regression analysis was performed to evaluate the association between eGFR and recanalization rate (thrombolysis in cerebral infarction scale $2 b-3$ ), symptomatic intracranial hemorrhage $(\mathrm{s} \mid \mathrm{CH})$, death in hospital, death at 3 months and poor functional outcome (modified Rankin Scale 3-6 at 3 months).

Results: A total of 373 patients were included in the study. Of them, 130 (34.9\%) patients were in the eGFR group $\mathrm{G} 1,170$ (45.6\%) in G2, 46 (12.3\%) in G3a, 27 (7.2\%) in G3b-5. In multivariable logistic regression analysis, reduced eGFR was associated with increased risk of sICH (G3a, $p=0.016)$ and 3-month death (G3b-5, $p=0.025)$. However, no significant effects were observed between reduced eGFR and the risk of recanalization rate $(p=0.855)$, death in hospital ( $p=0.970)$, and poor functional outcome $(p=0.644)$.

Conclusions: For AIS patients underwent MT, reduced eGFR was associated with increased risk of sICH and 3month death. However, there were no appreciable effects of reduced eGFR on recanalization rate, death in hospital and 3-month functional outcome.
\end{abstract}

Keywords: Estimated glomerular filtration, Mechanical thrombectomy, Outcomes, Stroke

\section{Background}

Chronic kidney disease (CKD) is an increasing global public health issue. Worldwide, the prevalence of CKD is estimated to be $8-16 \%$ [1]. In China, it constitutes $4.5 \%$ of all hospitalizations [2].

\footnotetext{
*Correspondence: zhjsh333@126.com

${ }^{\dagger}$ Jie Yang and Junshan Zhou contributed equally to this work.

${ }^{2}$ Department of Neurology, Nanjing First Hospital, Nanjing Medical University, Nanjing, China

Full list of author information is available at the end of the article
}

CKD is an independent risk factor of cardiovascular disease [3]. For stroke patients, some studies suggested that CKD with decreased estimated glomerular filtration rate (eGFR) significantly increased the risk of stroke mortality $[4,5]$. In intravenous thrombolysis (IVT) therapy, one study with large sample size revealed that low GFR was independently associated with poor outcome and the risk of symptomatic intracerebral hemorrhage (sICH) [6]. However, the results of other studies were conflicting $[7,8]$. 
Besides IVT, mechanical thrombectomy (MT) recently has been proven to be an effective therapy for acute ischemic stroke (AIS) [9]. In patients with 6 to $24 \mathrm{~h}$ after onset, MT is the only reperfusion strategy for the selected patients [10]. However, during the MT procedure, over $90 \%$ iodinated contrast media $(\mathrm{CM})$ is eliminated by the kidney [11]. Renal dysfunction can cause the accumulation of $\mathrm{CM}$ in blood plasma and increase its nephrotoxicity. Thus, compared with other AIS treatment, in MT procedure, patients with reduced eGFR are at a higher risk of acute kidney injury (AKI), which increases the hospital mortality $[12,13]$. Up to present, the relationship between renal function and outcomes after MT has not been sufficiently evaluated.

In this study, we aimed to investigate the influence of renal function on outcomes of MT and therefore provide information to improve patient selection for MT.

\section{Methods}

\section{Study design and patients}

Nanjing First Hospital Stroke Registry is a prospective cohort study. It was designed to achieve comprehensive information of stroke patients in Nanjing, China. The study protocol was approved by the hospital ethics committee. Form April 2015 to February 2019, all consecutive AIS patients receiving MT were included in the study. The patient's inclusion criteria for MT were in accordance with national guideline recommendation and neuro-interventionist's evaluation. The patients also received subsequent standard treatment for AIS. Written informed consent was obtained from each patient or their statutory.

\section{Data collection}

On admission, baseline characteristics were recorded including demographic characteristics, past medical history, disease severity evaluation, vital signs and crucial laboratory tests [glucose, platelet count, serum creatinine, prothrombin time and international normalized ratio (PT/INR)]. Other laboratory tests (lipid panel, liver function panel) were obtained during hospitalization. Magnetic resonance imaging (MRI) was performed before the MT procedure. Computerized tomographic scan (CT) was performed at $24 \mathrm{~h}$ after MT or anytime if neurological deterioration was observed. At 3 months after discharge, patients were evaluated by the telephone follow-up.

\section{Renal function}

Serum creatinine was measured on admission. eGFR was evaluated by the CKD Epidemiology Collaboration (CKD-EPI) equation [14]. According to the guideline [15], renal function was categorized into G1 $(\geq 90 \mathrm{ml} /$ $\left.\mathrm{min} / 1.73 \mathrm{~m}^{2}\right)$, G2 $\left(60-89 \mathrm{ml} / \mathrm{min} / 1.73 \mathrm{~m}^{2}\right)$, G3a $(45-59$ $\left.\mathrm{ml} / \mathrm{min} / 1.73 \mathrm{~m}^{2}\right)$, G3b $\left(33-44 \mathrm{ml} / \mathrm{min} / 1.73 \mathrm{~m}^{2}\right)$, G4 (15$\left.29 \mathrm{ml} / \mathrm{min} / 1.73 \mathrm{~m}^{2}\right)$ and $\mathrm{G} 5\left(<15 \mathrm{ml} / \mathrm{min} / 1.73 \mathrm{~m}^{2}\right)$. reduced eGFR was defined as GFR $<90 \mathrm{ml} / \mathrm{min} / 1.73 \mathrm{~m}^{2}$ (G2-5). Renal dysfunction was defined as GFR $<60 \mathrm{ml} /$ $\mathrm{min} / 1.73 \mathrm{~m}^{2}$ (G3a-5). eGFR $\geq 90 \mathrm{ml} / \mathrm{min} / 1.73 \mathrm{~m}^{2}$ (G1) was the reference group, eGFR $\leq 44 \mathrm{ml} / \mathrm{min} / 1.73 \mathrm{~m}^{2}$ (G3b-5) were pooled together due to the small sample size.

\section{Outcome assessment}

The outcome measures were (1) recanalization rate, defined as thrombolysis in cerebral infarction scale (TICI) 2b-3; (2) sICH, defined as hemorrhage on CT scan with neurological deterioration of $\geq 4$ points on National Institutes of Health Stroke Scale (NIHSS) score [16]; (3) death in hospital; (4) death at 3 months; (5) long-term functional outcome, measured by modified Rankin Scale (mRS) at 3 months. Poor functional outcome was defined as mRS 3-6.

\section{Statistical analysis}

Continuous variables were presented as mean with standard deviation (SD) or median with inter-quartile range (IQR) when appropriate. Categorical variables were presented as numbers with percentages. First, we compared baseline characteristics across the 4 eGFR groups. We used flowing methods: for continuous variables, we used one-way ANOVA test or Kruskal-Wallis test; for categorical variables, we used chi-square test or Fisher's exact test. Then we conducted an univariable analysis using binary logistic regression models to evaluate the association between each clinical characteristic and each clinical outcome, in which eGFR was presented as a continuous variable. In multivariable logistic regression analysis, eGFR was categorized into 4 groups [G1 (reference group), G2, G3a, and G3b-5]. The following covariates were adjusted in the multivariable models: (1) variables with $p<0.1$ in the univariable analysis (2) factors identified by previous studies which are clinically relevant to the outcomes. We performed two models: model 1 was adjusted for age and sex. Model 2 was adjusted for all the potential confounders. SPSS (version 22.0 for Windows; IBM Inc., Armonk, NY, USA) was used for statistical analysis. $p<0.05$ was considered significant.

\section{Results}

Baseline clinical characteristics

A total of 374 AIS patients received MT treatment from April 2015 to February 2019, 1 were excluded due to lack of serum creatinine value $(n=373)$. At 3 months, 15 patients were lost to follow-up. Among eligible patients, $130(34.9 \%)$ patients were in the eGFR group G1 $\left(\geq 90 \mathrm{ml} / \mathrm{min} / 1.73 \mathrm{~m}^{2}\right), 170(45.6 \%)$ 
were in $\mathrm{G} 2\left(60-89 \mathrm{ml} / \mathrm{min} / 1.73 \mathrm{~m}^{2}\right), 46(12.3 \%)$ were in G3a $\left(45-59 \mathrm{ml} / \mathrm{min} / 1.73 \mathrm{~m}^{2}\right)$ and $27(7.2 \%)$ were in G3b-5 $\left(\leq 44 \mathrm{ml} / \mathrm{min} / 1.73 \mathrm{~m}^{2}\right)$. Baseline characteristics are presented in Table 1 . Patients with reduced eGFR were older and more commonly had hypertension, coronary artery disease, atrial fibrillation, previous TIA/stroke and higher NIHSS score. They were more likely to have higher PT/INR and glucose level. They also had lower total cholesterol and LDL levels. In addition, our study showed that patients with low GFR were more prone to have cardioembolism as stroke etiology $(p=0.013)$ whilst patients with higher GFR were large artery atherosclerosis $(p=0.047)$ (Table 1).

\section{Univariable analysis}

In univariable analysis, as continuous variable, reduced eGFR (per $10 \mathrm{ml} / \mathrm{min} / 1.73 \mathrm{~m}^{2}$ decrease) was associated with increased risk of $\mathrm{sICH}(p=0.001)$, death in hospital $(p=0.01)$, death at 3 months $(p<0.001)$ and poor functional outcome $(p<0.001)$, but it was not associated with recanalization rate $(p=0.51)$ (Supplemental Table 1$)$.

In unadjusted analysis, as categorical variable, compared to normal eGFR (G1), reduced eGFR was associated with increased risk of $\operatorname{siCH}(p=0.021)$, death at 3 months $(p=0.008)$ and poor functional outcome $(p<$ $0.001)$, but it was not associated with recanalization rate $(p=0.506)$ or death in hospital $(p=0.343)$ (Table 2$)$.

\section{Multivariable analysis}

After adjusting for age and sex (Model 1), compared to normal eGFR (G1), reduced eGFR was associated with a higher risk of $\mathrm{sICH}(\mathrm{G} 3 \mathrm{a}, p=0.01)$ and death at 3 months (G2, $p=0.04$; G3b-5, $p=0.023$ ) (Table 2).

In multivariable logistic regression analysis (Model 2), we adjusted all the potential confounders. Compared to normal eGFR (G1), reduced eGFR was still associated with increased risk of sICH (G3a, $p=0.016)$ and death at 3 months (G3b-5, $p=0.025)$. However, it was not associated with a higher risk of death in hospital $(p=0.970)$ or poor functional outcome $(p=0.644)$. The recanalization rate was not influenced by renal function $(p=0.855)$ (Table 2).

\section{Discussion}

Our study, which included 373 AIS patients, evaluated the influence of renal function on outcomes after MT. The results revealed that in short-term outcomes, compared with normal eGFR (G1), reduced eGFR was not associated with recanalization rate and death in hospital. Renal dysfunction G3a $\left(45-59 \mathrm{~mL} / \mathrm{min} / 1.73 \mathrm{~m}^{2}\right)$ increased the risk of sICH. In long-term outcomes, renal dysfunction G3b-5 $\left(\leq 44 \mathrm{ml} / \mathrm{min} / 1.73 \mathrm{~m}^{2}\right)$ increased the risk of death at 3 months. However, there was no appreciable effect of reduced eGFR on functional outcome at 3 months.

Several previous observational studies investigated the relationship between renal function and stroke outcomes. However, only one study included MT patients and their results were conflicting [4-8, 17-21]. Some studies found that reduced eGFR significantly increased the risk of stroke mortality $[4,5]$ and it was associated with poor outcome and sICH after intravenous thrombolysis [6]. However, other studies found such influence was not statistically significant $[7,8]$. These discrepancies may be explained by the following reasons. First, the stroke type, some studies included both ischemic and hemorrhagic stroke $[5,7,18,19]$, while others were one type only $[4,6,8,17,20,21]$. Second, different onset time. Some study included the stroke patients within 24 h of onset $[6,8,18,21]$, while others were 14 days [4] or 7 days [5]. Third, the difference of standard treatment. Some studies were conducted 10 years ago [14, 16-20]. The standard treatment recommended by guidelines were updated during this period, which may cause the different outcomes.

Unlike other AIS treatment, in MT, the risk of contrast-induced nephropathy (CIN) was increased with reduced eGFR. It was associated with increased morbidity, hospital stay and mortality [13]. There was only one previous study conducted by Laible et al. investigated the renal function and MT outcomes [21].

In short-term outcomes, our study found eGFR 45-59 $\mathrm{ml} / \mathrm{min} / 1.73 \mathrm{~m}^{2}$ (G3a) was associated with the risk of sICH. However, the study of Laible did not observe any relationship between renal dysfunction (eGFR $<60 \mathrm{ml} /$ $\min / 1.73 \mathrm{~m}^{2}$ ) and $\mathrm{sICH}$ or any ICH. In the study of Laible et al. with German population, the overall sICH rate was $6.8 \%$. It was significantly lower than our study with Chinese population (10.7\%). In another Chinese study evaluating $\mathrm{sICH}$ after $\mathrm{MT}$, the $\mathrm{sICH}$ rate was even higher at about 16\% [22]. Not only MT, in intravenous thrombolysis therapy, Asian population also had a significantly higher risk of sICH compared with the white group [23]. These results indicated that race/ethnic may cause the difference in bleeding risk after MT. Thus, we suggested that Chinese/Asian patients should be carefully monitored during and after MT procedure.

In long-term outcomes of 3 months mortality and poor outcome (mRS 3-6), our results were in accordance with Laible et al. In Laible et al., as a continuous variable, eGFR (each decrease of $10 \mathrm{ml} / \mathrm{min} / 1.73 \mathrm{~m}^{2}$ ) was associated with increased risk of mortality while as categorical variable, our study showed the group of eGFR $\leq 44 \mathrm{ml} / \mathrm{min} / 1.73 \mathrm{~m}^{2}$ (G3b-5) increased such risk. However, both our two studies observed that even though the mortality risk was increased, the functional outcome seemed not to be influenced by renal function. The 
Table 1 Baseline characteristics of patients according to eGRF

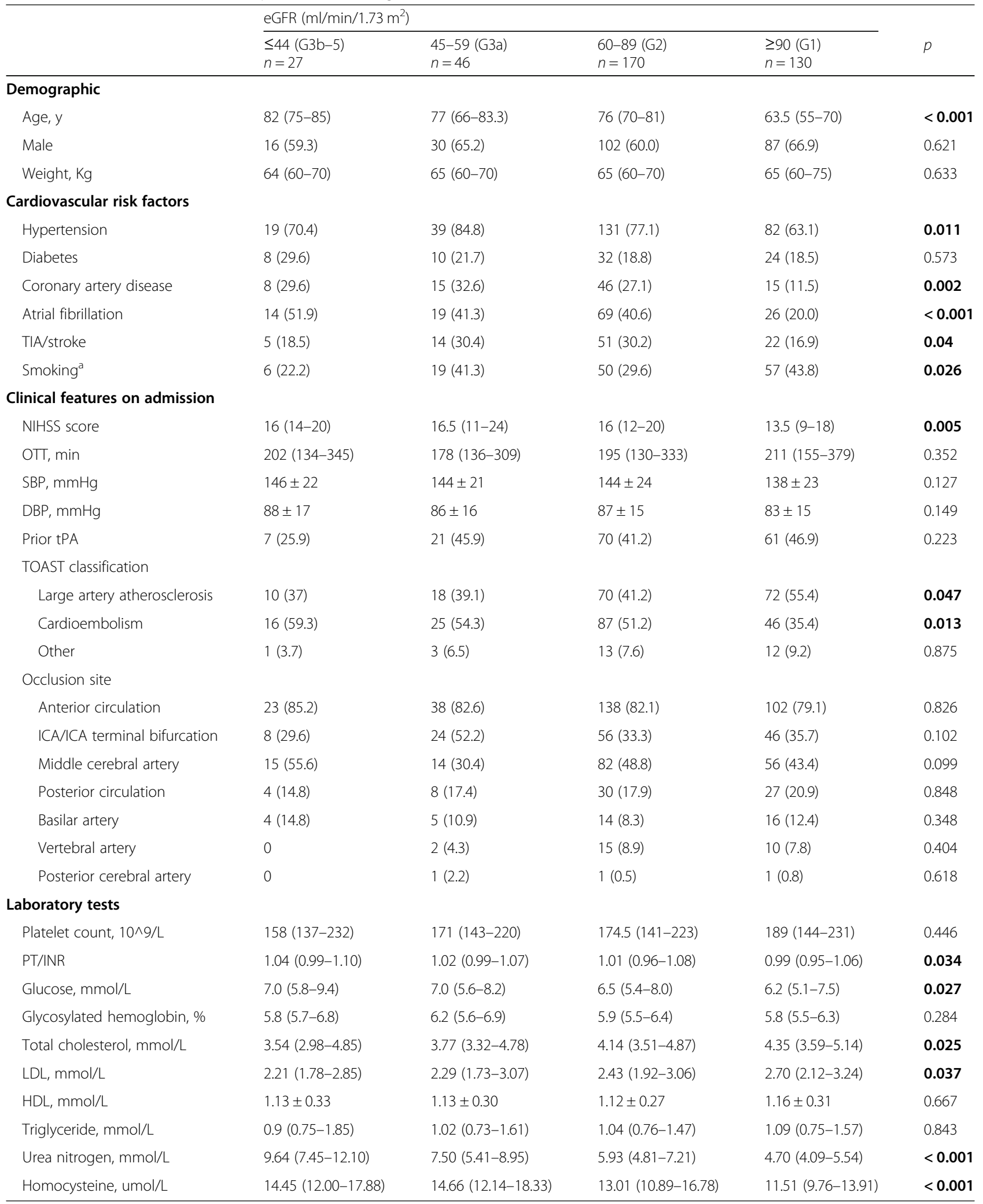

TIA transient ischemic attack, NIHSS National Institutes of Health Stroke Scale, OT, onset to treatment time, SBP systolic blood pressure, DBP diastolic blood pressure, tPA tissue plasminogen activator, ICA internal carotid artery, PT/INR prothrombin time and international normalized ratio, LDL low density lipoprotein $H D L$ high density lipoprotein

${ }^{\mathrm{a}}$ Smoking status including both former and current 
Table 2 Multivariable analysis of outcomes according to the eGFR

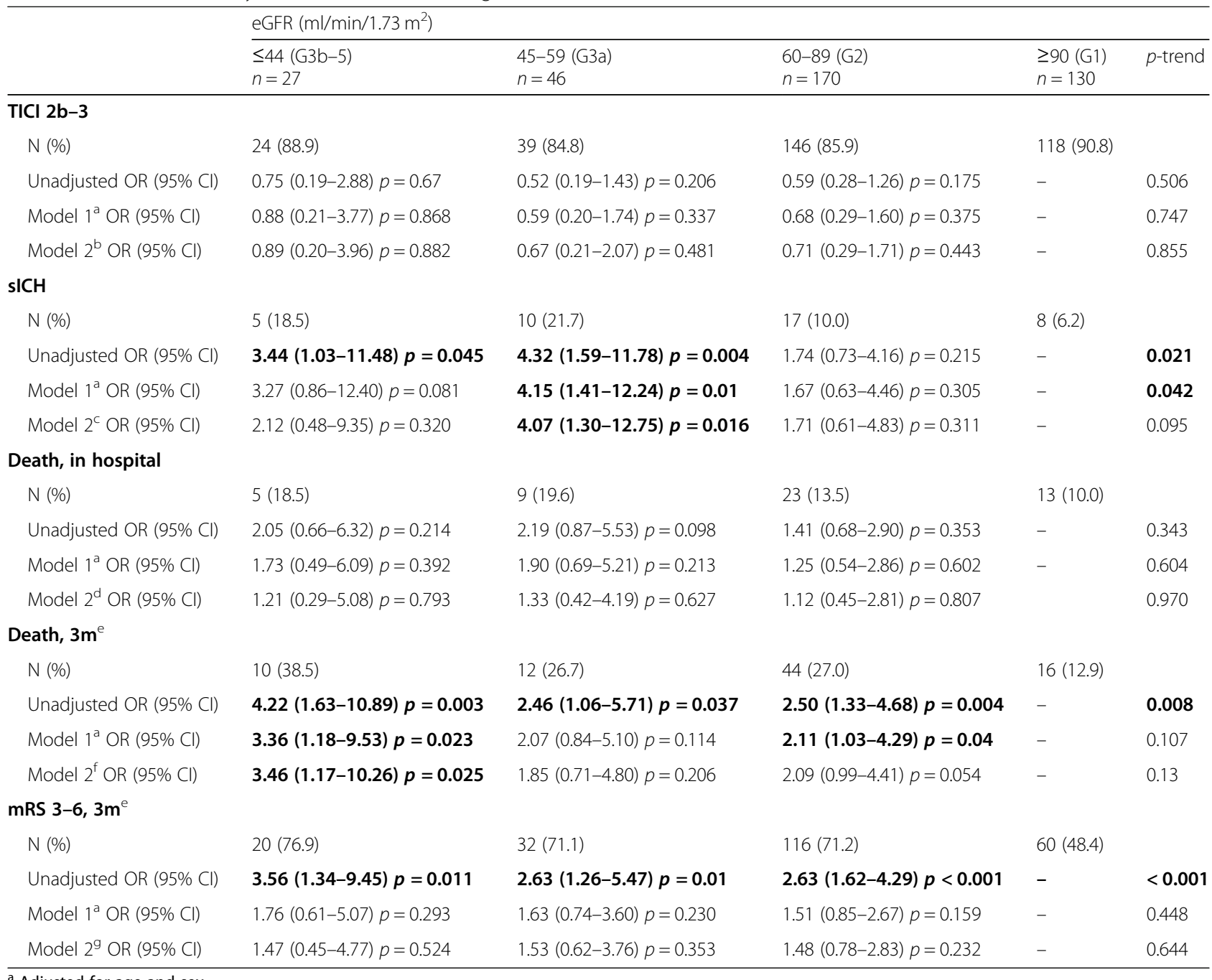

${ }^{a}$ Adjusted for age and sex

${ }^{b}$ Adjusted for age, sex, NIHSS, OTT and glucose

c Adjusted for age, sex, NIHSS, OTT, previous TIA/stroke, SBP and glucose

${ }^{d}$ Adjusted for age, sex, NIHSS, OTT, coronary artery disease, PT/INR and glucose

e 15 patients were lost to follow-up. 6 in G1, 7 in G2, 1 in G3a, 1 in G3b-5

${ }^{\mathrm{f}}$ Adjusted for age, sex, NIHSS, OTT and glucose

${ }^{g}$ Adjusted for age, sex, NIHSS, OTT, atrial fibrillation, smoking, glucose and HDL

plausible explanation of this result is still unidentified. But we presumed that patient with reduced eGFR had more comorbidities such as mineral and bone disorder, malnutrition and anemia. These comorbidities may not directly influence the neurological outcomes but it could increase the risk of death in elderly population.

\section{Limitations}

There were some limitations in our study. First, our study was a single-center observational cohort with only Chinese patients, the results may not be applicable to other populations. Second, CKD-EPI equation using creatinine has been proved to be more accurate than MDRD equation and has been recommended by current guidelines $[15,24]$. However, in our study, the mean age of the patient was 71.2 years. The equation combined with both creatinine and cystatin $\mathrm{C}$ may be more accurate than using creatinine alone for elderly individuals [25].

Despite the limitations, our study revealed that reduced eGFR did not represent a risk factor for recanalization rate, death in hospital and poor functional outcome. Renal dysfunction may not be considered as an exclusion criteria for MT.

\section{Conclusions}

Our study suggested that in AIS patients who underwent MT, reduced eGFR was associated with increased risks of sICH and death at 3 months. However, there were no appreciable effects of reduced eGFR on recanalization rate, death in hospital and functional outcome at 3 months. 


\section{Supplementary information}

Supplementary information accompanies this paper at https://doi.org/10. 1186/s12883-020-01720-5.

Additional file 1: Table S1. Univariable analysis of clinical characteristics in patients treated with EVT.

\section{Abbreviations}

CKD: Chronic kidney disease; eGFR: Estimated glomerular filtration rate; IVT: Intravenous thrombolysis; sICH: Symptomatic intracerebral hemorrhage; MT: Mechanical thrombectomy; CM: lodinated contrast media; AKI: Acute kidney injury; PT/INR: Prothrombin time and international normalized ratio; MRI: Magnetic resonance imaging; CT: Computerized tomographic scan; CKD-EPI: CKD Epidemiology Collaboration Equation; TICl: Thrombolysis in Cerebral Infarction Scale; NIHSS: National Institutes of Health Stroke Scale; mRS: Modified Rankin Scale; SD: Standard deviation; IQR: Inter-quartile range; CIN: Contrast-induced nephropathy

\section{Acknowledgements}

Not applicable.

\section{Authors' contributions}

XP planned and conducted the research, reported the data and was responsible for writing and editing the manuscript. FZ and RS collected the data. HA revised the statistical analysis and the manuscript. JY, YZ and JZ supervised the conduction of the research. All authors have read and approved the manuscript.

\section{Funding}

This report is independent research partially funded by Nanjing Pharmaceutical Association. The study was designed, conducted, analyzed and interpreted by the investigators independent of sponsors.

\section{Availability of data and materials}

All data have been presented within the manuscript and additional supporting files.

\section{Ethics approval and consent to participate}

The study protocol was approved by Nanjing First hospital Ethics Committee. Written informed consent was obtained from each patient or statutory agent.

\section{Consent for publication}

Not applicable.

\section{Competing interests}

The authors declare that they have no competing interests.

\section{Author details}

'Department of Pharmacy, Nanjing First Hospital, Nanjing Medical University, Nanjing, China. ${ }^{2}$ Department of Neurology, Nanjing First Hospital, Nanjing Medical University, Nanjing, China. ${ }^{3}$ Department of Preventive Medicine and Public Health, Faculty of Medicine, Fukuoka University, Fukuoka, Japan. ${ }^{4}$ Department of Neurology, The First Affiliated Hospital of Chengdu Medical College, Chengdu 610500, China.

Received: 21 January 2020 Accepted: 7 April 2020

\section{Published online: 14 April 2020}

\section{References}

1. Vivekanand J, Guillermo GG, Kunitoshi I, et al. Chronic kidney disease: global dimension and perspectives. Lancet. 2013;382:260-72.

2. Zhang L, Wang H, Long J, et al. China kidney disease network (CK-NET) 2014 annual data report. Am J Kidney Dis. 2016:68:A4.

3. Ene-lordache B, Perico N, Bikbov B, et al. Chronic kidney disease and cardiovascular risk in six regions of the world (ISN-KDDC): a cross-sectional study. Lancet Glob Health. 2016;4:e307-19.

4. Xianwei W, Yilong W, Chunxue W, et al. Association between estimated glomerular filtration rate and clinical outcomes in patients with acute ischaemic stroke: results from China National Stroke Registry. Age Ageing. 2014:43:839-45.

5. Nugroho AW, Arima H, Miyazawa I, et al. The association between glomerular filtration rate estimated on admission and acute stroke outcome: the Shiga stroke registry. J Atheroscler Thromb 2018; 25: 570-579.

6. Gensicke H, SM Zinkstok, YB Roos, et al. IV thrombolysis and renal function Neurology 2014; 83: 1583-1583.

7. Yang J, Arima H, Zhou J, et al. Effects of low estimated glomerular filtration rate on outcomes after stroke: a hospital-based stroke registry in China. Eur J Neurol. 2014;21:1143-5.

8. Hsieh CY, Lin HJ, Sung SF, et al. Is renal dysfunction associated with adverse stroke outcome after thrombolytic therapy? Cerebrovasc Dis. 2014;37:51-6.

9. Powers WJ, Rabinstein AA, Ackerson T, et al. 2018 guidelines for the early Management of Patients with Acute Ischemic Stroke: A guideline for healthcare professionals from the American Heart Association/American Stroke Association. Stroke. 2018;49:e46-e110.

10. Nogueira RG, Jadhav AP, Haussen DC, et al. Thrombectomy 6 to 24 hours after stroke with a mismatch between deficit and infarct. N Engl J Med. 2018;378:11-21.

11. Hogstrom B, Ikei N. Physicochemical properties of radiographic contrast media, potential nephrotoxicity and prophylaxis. Clin Exp Pharmacol Physiol. 2015:42:1251-7.

12. Chaudery $H$, MacDonald N, Ahmad T, et al. Acute kidney injury and risk of death after elective surgery: prospective analysis of data from an international cohort study. Anesth Analg. 2019;128:1022-9.

13. Mamoulakis C, Tsarouhas K, Fragkiadoulaki I, et al. Contrast-induced nephropathy: basic concepts, pathophysiological implications and prevention strategies. Pharmacol Ther. 2017;180:99-112.

14. Levey AS, Stevens LA, Schmid CH, et al. A new equation to estimate glomerular filtration rate. Ann Intern Med. 2009;150:604-12

15. Stevens PE, Levin A. Kidney disease: improving global outcomes chronic kidney disease guideline development work group members. Evaluation and management of chronic kidney disease: synopsis of the kidney disease: improving global outcomes 2012 clinical practice guideline. Ann Intern Med. 2013:158:825-30.

16. Torbey MT, Jauch E, Liebeskind DS, et al. Thrombolysis 3 to 4.5 hours after acute ischemic stroke. N Engl J Med. 2008:359:2839.

17. Lyrer PA, Fluri F, Gisler D, et al. Renal function and outcome among stroke patients treated with IV thrombolysis. Neurology. 2008;71:1548-50.

18. Tsagalis $G$, Akrivos T, Alevizaki M, et al. Renal dysfunction in acute stroke: an independent predictor of long-term all combined vascular events and overall mortality. Nephrol Dial Transpl. 2009:24:194-200.

19. Yahalom G, Schwartz R, Schwammenthal Y, et al. Chronic kidney disease and clinical outcome in patients with acute stroke. Stroke. 2009:40:1296303.

20. Mostofsky E, Wellenius GA, Noheria A, et al. Renal function predicts survival in patients with acute ischemic stroke. Cerebrovasc Dis. 2009;28:88-94.

21. Laible M, Mohlenbruch MA, Pfaff J, et al. Influence of renal function on treatment results after stroke thrombectomy. Cerebrovasc Dis. 2017;44:351-8.

22. Hao $Y$, Yang $D$, Wang $H$, et al. Predictors for symptomatic intracranial hemorrhage after endovascular treatment of acute ischemic stroke. Stroke. 2017:48:1203-9.

23. Mehta $\mathrm{RH}$, Cox M, Smith EE, et al. Race/ethnic differences in the risk of hemorrhagic complications among patients with ischemic stroke receiving thrombolytic therapy. Stroke. 2014:45:2263-9.

24. Jalalonmuhali M, Lim SK, Shah MNM, et al. MDRD vs. CKD-EPI in comparison to (51) Chromium EDTA: a cross sectional study of Malaysian CKD cohort. BMC Nephrol. 2017;18:363

25. Fan L, Levey AS, Gudnason V, et al. Comparing GFR estimating equations using Cystatin C and Creatinine in elderly individuals. J Am Soc Nephrol. 2015:26:1982-9.

\section{Publisher's Note}

Springer Nature remains neutral with regard to jurisdictional claims in published maps and institutional affiliations. 